\title{
The selection of homograph meaning: Word association when context changes
}

\author{
DAVID S. GORFEIN \\ University of Texas, Arlington, Texas \\ STEPHANIE BERGER \\ College of Mount St. Vincent, Riverdale, New York \\ and \\ ANDREA BUBKA \\ St. Peter's College, Jersey City, New Jersey
}

\begin{abstract}
In a study of lexical ambiguity processing, responses to homographs were examined in a word association task. The context of repeated exposures of a homograph was manipulated by requiring a response to a word related to a meaning of the homograph on the trial prior to homograph presentation. A change of that relationship reduced the effectiveness of the contextual item as a prime on the second occurrence of the homograph. In response to a third unprimed occurrence of the homograph, associations were consistent with a conclusion that when semantic contexts are opposed, a "primacy effect" is obtained. The overall effects in the studies reported are seen as consistent with the theoretical view of Simpson and Kang (1994) that processing and responding to one meaning of a homograph result in the inhibition of alternative meanings. A mechanism to account for that inhibition is proposed.
\end{abstract}

In 1976, Schvaneveldt, Meyer, and Becker demonstrated that the effectiveness of a homograph as a related prime depended on the context that preceded the homograph. When a homograph was preceded by a word that was related to the same meaning as was the subsequent target (e.g., SAVE-BANK-MONEY), responses were faster than responses to homographs preceded by an unrelated word (e.g., DAY-BANK-MONEY). These in turn were faster than responses to triples containing a prime related to the other meaning of the homograph (e.g., RIVER-BANK-MONEY). Subsequently, Onifer and Swinney (1981) reported that homographs embedded in auditory disambiguating sentences primed lexical decisions to visually presented words related to either of the homograph's meanings. However, if the target was delayed for $1.5 \mathrm{sec}$, only the contextappropriate meaning of the homograph was primed. Several theorists have proposed that the selection of one meaning of a homograph leads to the immediate active suppression of alternative meanings (Gernsbacher, 1990; Neill, 1989).

Krueger (1990) found that processing the second of two sentences containing the same homograph in alternate

We thank Christian DeBiasi, Susan DeGuilio, and Sara Jamshidi, who served as experimenters. Three reviewers of an earlier version of this manuscript, Doug Nelson, Dani McKinney, and Greg Simpson, provided thoughtful ideas that resulted in clarification of the ideas presented. We are grateful to them, as well as to Jim Erickson, for their critical reviews. Correspondence should be addressed to D. S. Gorfein, Department of Psychology, University of Texas, Box 19528, Arlington, TX 76019-0528 (e-mail: gorfein@utarlg.uta.edu) meaning contexts was slower than processing following a control sentence. Simpson and Kang (1994) determined that a homograph that had primed the naming of a word related to one of its meanings actually slowed performance on a subsequent word related to an alternate meaning. On the basis of their extensive review of homograph research, they concluded, "Processing one meaning of a homograph and responding to that meaning results in the active and specific inhibition of competing meanings" (Simpson \& Kang, 1994, p. 376).

In the present investigation, we tested whether the inhibition of competing meanings observed by Simpson and his colleagues in reaction time studies extended to the discrete response selection required by the word association test (WAT). The WAT enabled us to examine whether priming a homograph in the direction of one of its meanings makes associates related to its alternative meaning less available. Using the WAT allowed us to evaluate the residual effect of responding to a homograph in two different contexts on responding to that homograph in a subsequent neutral context.

Early studies of priming in the WAT (Clifton, 1966; Cofer, 1967; Cramer, 1968; Segal, 1967; Segal \& Cofer, 1960; Storms, 1958) defined priming on the basis of a specific preselected associate of the word association stimulus. With the exception of Cramer, the preselected word was presented in an orienting task. An increase in response frequency of that word indicated priming. Segal reported that the likelihood that homographs initially embedded in an analogy test were produced on a subsequent WAT depended on whether the association stimulus was 
identical to, related to, or different from the meaning presented in the analogy. The word association stimulus RIVER elicited the response BANK with greatest frequency if the analogy had been "BANK is to RIVER," a lesser frequency if the analogy had been "BANK is to STREAM," and at near chance levels if the analogy had been "BANK is to MONEY."

Cramer (1968) presented homographs in the context of two words selected to guide the production of a particular response. Participants were told to read aloud the first two words on a trial (e.g., ANGER, FIGHT) and then produce a word association to a third word (TAP). Performance was scored for the response HIT. In another group, the word TAP was preceded by WHISKEY and ALE and the target word was BEER. Both contexts elicited their responses at greater than normative frequency.

In our studies priming is defined as an increase in the frequency of WAT responses that are related to a particular meaning of a homograph (e.g., responses related to the financial meaning of BANK). Cramer (1968) analyzed the increase in responses related to the primed meaning of the homograph (e.g., beverage-related responses to the stimulus TAP given the primes WHISKEY and ALE). She concluded that beyond an increase in the specific response BEER, the prime had little influence. However, Cramer's manipulation may have led participants to treat the task as a problem-solving task and use the $6 \mathrm{sec}$ provided to respond to find a common response word to WHISKEY, ALE, and TAP.

Berger, Bubka, and Gorfein (1990) reported an increase in responses from the primed meaning class of a homograph. Words from a meaning class were more likely to be elicited to a homograph when the previous trial required a response to a related word than when the prior response was to an unrelated word. Responding to LIVER on a WAT trial increased the frequency of responses such as HEART to the homograph ORGAN on the next trial.

The three experiments in this paper used a similar method of presenting homographs and primes in a WAT. The participant viewed a series of words and was instructed to write the first word to come to mind on each trial. An advantage of this method is that prime trials are not distinguished from homograph trials. This is in marked contrast to priming in the naming task and to typical single-trial priming events in lexical decision. It also differs from the priming technique employed in the WAT by Cramer (1968) in which the participant only read the primes and responded to the homograph.

In our experiments, each homograph initially followed a word related to one meaning and on a second occurrence was preceded by a word related to its alternative meaning. For example, ORGAN was presented on trial $n$ preceded by the related word LIVER on trial $n-1$. The word MUSIC occurred on trial $n+23$ and the homograph ORGAN again on trial $n+24$. This allowed us to test the hypothesis that the first occurrence of the homograph would result in a diminished effect of the second-occurrence prime as contrasted with the effect of a first-occurrence prime. We were also interested in whether the effect of earlier priming trials would carry over to trials where the homograph was presented in a neutral context. Continuing the previous example, we examined which response class would be elicited when ORGAN was presented on trial $n+64$ following an unrelated stimulus (HAT) on trial $n+63$. We also examined whether the effect on the neutral primed trials depended on the time between the homograph occurrences. Experiment 1 tested these conditions.

\section{EXPERIMENT 1}

\section{Method}

\section{Participants}

Thirty-two Adelphi University students participated in Experiment 1 as part of a requirement for an introductory psychology course. The participants in all experiments were native English speakers. Participants were assigned to list at random.

\section{Materials}

Twenty-eight homographs were selected from the Adelphi norms (Gorfein, Viviani, \& Leddo, 1982). Items were selected so that the two meanings of the homograph were reasonably balanced. The mean proportion of occurrences for the more common (dominant) meaning of the 28 homographs was .605 , with a range of .50-.72. We used homographs that had two distinct meanings, when all senses of a meaning were scored as the same meaning. Therefore the secondary meaning of the homograph had a normative proportion of .395. The mean frequency of occurrence per million of the homographs was 48.25 (Kučera \& Francis, 1967).

Two primes were chosen for each homograph, one related to its dominant meaning and the other to its secondary meaning. The choice of prime word was somewhat arbitrary since relatedness norms for the homographs did not exist at the time of list creation. It was our aim to select words that while related to the homographs chosen would be unlikely direct associates of the homograph, to minimize what Cofer (1967) called "direct priming." Across participants the same prime preceded a first and a second occurrence of a homograph equally often. We therefore compared the amount of priming obtained from the same prime when it preceded a first occurrence with priming when it preceded a second homograph occurrence. We look at a homograph MARCH following the prime DECEMBER when the participant has earlier responded to ARMY preceding MARCH and in the absence of the earlier experience. Because of the counterbalancing, the actual size of the relationship between any prime and homograph is largely irrelevant to the questions asked.

Materials were divided into four subsets matched on meaning dominance and were arranged into four unique word lists so that each homograph appeared in each condition of the experiment once. Two context conditions (mixed and single) were included in each list. In the mixed condition, 14 homographs were presented three times. These homographs were preceded by a prime related to one meaning on their first occurrence, by a prime related to the alternate meaning on second occurrence, and by a word unrelated to either meaning on third occurrence. In the single-prime condition, the remaining 14 homographs were presented twice, preceded by a related prime on the first occurrence and by a word unrelated to either meaning on the second occurrence. On first occurrence, half (7) of the homographs in each condition were preceded by a word related to their dominant meaning while the remaining homographs were preceded by a word related to their secondary meaning.

Each list was constructed in three parts. Part 1 (Trials 1-84) of each list included the first occurrence of the 14 homographs and their primes in the mixed-prime condition and the 14 homographs and 
primes in the single-prime condition, as well as 28 filler items. Filler words were unrelated to the homographs. A set of 12 filler items was also included between Parts 1 and 2 to ensure a minimum separation of first and second occurrences of homographs. Actual separation varied from 24 to 142 trials with a mean of 82 events.

Part 2 (Trials 97-166) included the second occurrences of the 14 mixed-prime homographs preceded by their alternate meaning prime. Homographs in the single-prime condition were replaced by unrelated words, which were preceded by the alternative meaning prime of each replaced homograph. Part 2 included 28 filler trials, with half of the fillers being repetitions of Part 1 filler words.

Part 3 followed Part 2 immediately or was delayed by 40 filler trials. Delay was manipulated between lists and resulted in Part 3 trials of either 167-206 or 207-262. The 56 trials of Part 3 included the third occurrences of the 14 mixed-prime homographs, 14 second occurrences of the single-prime homographs, and a third occurrence of 14 fillers, as well as a second occurrence of another 14 fillers.

Counterbalancing homographs across the meaning primed on first homograph occurrence (dominant or secondary), prime context conditions (mixed or single), and delay ( 0 or 40 trials) resulted in eight unique lists that were employed equally often in the experiment.

\section{Procedure}

Participants were tested individually on the WAT. Single words were presented centered on a computer screen, along with their trial numbers. Participants were instructed to write the first word each item brought to mind alongside the trial number on the answer sheet provided. No mention was made of primes or homographs. From the participant's standpoint, each word was a unique item in the WAT. After responding, the participant pressed the space bar and the next item appeared on the screen. If no response was made and $6 \mathrm{sec}$ elapsed, the next trial was presented. Participants were informed that some items would be repeated and were instructed that they should always write the first word that came to mind. The task occupied 20-25 $\mathrm{min}$.

\section{Results and Discussion}

A rater blind to the prime assigned the response to each homograph to a meaning class. When a response could not be readily assigned to one of the two meanings of a homograph, a second rater attempted to classify it. Omissions and responses that could not be scored were dropped from the analyses ( $2.1 \%$ of all responses). The reliability of scoring was measured by having two raters score 28 homograph trials from the Part 3 data of a random sample of 10 participants. Of the 280 total judgments of responses, 278 were in agreement $(99.3 \%)$.

The score in each condition was the proportion of scorable responses that were judged to be related to the dominant meaning of the homograph. Priming was defined as the difference between the chance value (.605) and the observed proportion of each condition. For the dominant prime, a priming score was the proportion of responses in the dominant direction ${ }^{1}$ minus .605. Priming for secondary primes was defined as .605 minus the proportion of dominant responses. The alpha level for all analyses was set at .05 in all experiments.

Table 1 reports the proportion of dominant meaning responses to the homographs as a function of the prime history in each part of the experiment as well as a priming score for each condition. Each of the eight priming
Table 1

Proportion of Dominant Meaning Word Association Responses (Score) and Observed Priming in Each Part of Experiment 1

\begin{tabular}{|c|c|c|c|}
\hline \multicolumn{2}{|c|}{ Prime History } & Score & Priming \\
\hline \multicolumn{4}{|c|}{ Part 1} \\
\hline Prior & Current & & \\
\hline - & Dominant & .766 & .162 \\
\hline - & Secondary & .427 & .178 \\
\hline \multicolumn{4}{|c|}{ Part 2} \\
\hline Prior & Current & & \\
\hline Secondary & Dominant & .725 & .120 \\
\hline Dominant & Secondary & .504 & .101 \\
\hline \multicolumn{4}{|c|}{ Part 3 Unprimed } \\
\hline Part 1 & Part 2 & & \\
\hline Dominant & - & .692 & $.087^{*}$ \\
\hline Secondary & - & .518 & .101 \\
\hline Dominant & Secondary & .648 & .043 \\
\hline Secondary & Dominant & .542 & .063 \\
\hline
\end{tabular}

Note-Priming is the deviation from the normative baseline of .615 . *Priming in Part 3 is defined with respect to the Part 1 prime for all conditions.

scores was significantly greater than zero priming by a two-tailed $t$ test. This indicated that our method, which defined priming in the WAT in relation to the meaning class of the response, produced results similar to those of earlier studies that defined priming with respect to specific response targets (Cramer, 1968; Segal, 1967).

The nature of the design necessitated several analyses of variance (ANOVAs). An ANOVA ${ }^{2}$ of Part 1 indicated that the observed proportion of dominant responses was significantly different when the homograph was preceded by a prime trial related to the dominant meaning (.767) than when the prime trial item was related to the secondary meaning [.427, $\left.F(1,30)=165.17, M S_{\mathrm{e}}=0.0112\right]$.

All homographs in Part 2 were in the mixed-context condition and so were primed with the meaning alternate to that primed in Part 1. The proportion of dominant responses to each of the Part 2 primes differed significantly $\left[F(1,30)=18.26, M S_{\mathrm{e}}=0.0424\right]$, with the proportion of dominant responses when the prime was dominant (.725) being greater than when the prime was secondary (.504). The magnitude of priming in Parts 1 and 2 was compared in an ANOVA of the priming scores (Part 1 vs. Part 2, by current prime). The mean priming effect in Part $1(.170)$ was significantly different from the mean priming effect in Part $2\left[.121, F(1,60)=5.32, M S_{\mathrm{e}}=\right.$ $0.0216]$. These results indicate a reduction in the effectiveness of a prime of one homograph meaning given prior exposure to an alternate meaning prime. This finding is consistent with the loss of prime effectiveness in word naming (Simpson \& Kang, 1994; Simpson \& Kellas, 1989). The implications of these results are addressed in the General Discussion.

Part 3 tested how the prime history of a homograph influenced subsequent responses to that homograph in a neutral context. Priming in Part 3 was defined as the proportion of responses that were related to the meaning 
primed in Part 1. We then compared the amount of priming in Part 3 to the amount in Part 1. A 2 prime type (dominant, secondary) $\times 3$ prime history (Part 1 , Part 3 single, Part 3 mixed context) $\times 2$ delay ( 0 or 40 items between Parts 2 and 3) mixed-model ANOVA was performed. Neither the effect of delay $[F(1,30)<1]$ nor any of its interactions approached significance $(F<1$, in all cases). The effect of prime history was significant $[F(2,60)=$ $\left.11.10, M S_{\mathrm{e}}=0.0279\right]$. A post hoc analysis employing Tukey's procedure for pairwise comparisons (Myers \& Well, 1991, pp. 84-86) indicated that the higher proportion of primed responses in Part $1(.170)$ was significantly different from the priming observed in Part 3 to items in the single-prime condition (.089) or to those in the mixed prime history condition (.053). The difference within Phase 3 was not significant. However, the amount of priming in the single-prime condition $[t(31)=4.61]$ and the mixed-prime condition $[t(31)=2.39]$ was significant in the direction of the Part 1 meaning. The effect in the mixed condition is remarkable in that not only was there a departure from the more recent Part 2 prime, but also there was a significant "primacy effect." This effect was not unlike the classical effect of primacy in trait descriptions in the social psychological literature (see Anderson, 1996). The results were also consistent with results we have reported elsewhere for both spelling and word association responses to nonhomographic homophones (Gorfein \& Walters, 1989; Walters, 1988).

\section{EXPERIMENT 2}

Experiment 1 established the effect of the initial processing of a homograph on selecting an alternate meaning of that homograph in a new context. Further, processing homographs in two different contexts prior to a test in a neutral context resulted in a primacy effect. In the singleprime condition, responses to homographs in a neutral context showed a return toward the normative baseline.

In Experiment 2, we attempted to characterize these findings more precisely. The interval between first and second occurrences of homographs in the mixed-prime condition was large and variable in Experiment 1. In Experiment 2, the separation between first and second occurrences of homographs was fixed at either 10 or 30 items. A control condition was added in which previously primed homographs were presented in neutral contexts at the same lags as the first two occurrences in the mixedprime condition. This allowed a comparison of priming in the mixed-context condition to control items with similar priming history.

We reexamined the delay between the alternate meaning context in Part 2 and the neutral context in Part 3 in the mixed-prime condition. We hypothesized that as the delay between the second and third occurrences increased, the primacy effect would become stronger due to a loss in effectiveness of the most recent prime. We saw this as similar to the loss of recency effects in free recall over a filled interval (see, e.g. Glanzer \& Cunitz, 1966).
In Experiment 1, we manipulated type of prime (dominant, secondary) as a within-subjects condition. In Experiment 2 , the meaning of the first-occurrence prime was manipulated as a between-groups variable; one experimental group was assigned dominant primes and the other group secondary primes.

\section{Method}

\section{Participants}

Forty-eight Adelphi University students and 64 students at the University of Texas at Arlington served in this experiment to fulfill a course requirement. Participants were assigned to the four experimental conditions in a counterbalanced manner. Each of the four between-groups conditions included an equal proportion of the samples drawn from the two universities.

\section{Materials}

We created lists with the 28 homographs and the 56 prime words from Experiment 1. Four basic list types were constructed by manipulating lag between the first and second occurrences of a homograph ( 10 or 30 trials) as a between-lists variable and delay between the second and third occurrences ( 3 or 20 trials) as a within-lists factor.

In one set all 28 homographs were primed in the dominant direction on their first occurrence and in another set of lists, items were primed in the secondary direction. On the second occurrence, half of the items on each list were primed by their alternate meaning prime (mixed-context condition) and half by the unrelated alternate prime of another homograph (unrelated-prime control condition). Counterbalancing to ensure that every homograph appeared in each condition resulted in 16 different lists.

Each list consisted of 222 trials. Homograph sets were interwoven in the list. Therefore the second and third occurrences of some homographs preceded the first occurrence of others. Filler items, half of which were repeated at varying intervals, occupied 82 trial positions.

\section{Procedure}

The procedure and instructions were identical to those of Experiment 1 except that a fixed 6-sec trial was employed.

The design of the study was a mixed model. Lag between first and second occurrences (10 or 30 items) and Part 1 prime meaning (dominant, secondary) were between-groups variables. Contextual history (mixed or control) and delay between second and third occurrences of a homograph ( 3 or 20 trials) were within-groups variables. The task required $23 \mathrm{~min}$ to complete.

\section{Results and Discussion}

The proportion of scorable responses in the direction of the normative dominant meaning of the homograph was used for all conditions.

We collapsed the third-occurrence data across the delay intervals between second and third occurrences and reserved this delay variable for a separate ANOVA. A 3 (1st, 2 nd, or 3 rd occurrence) $\times 2$ (dominant or secondary prime on first occurrence) $\times 2$ (prime history, mixed or control) $\times 2$ (lag, 10 or 30 trials between 1 st and 2 nd homograph presentation) mixed model ANOVA was performed.

The lag between first and second homograph occurrences did not produce a significant main effect and did not enter into any interaction. Table 2 presents the proportion of responses scored as dominant meaning responses to the homograph collapsed over lag. Since, in this experiment, the primes were not equated for rela- 
Table 2

Proportion of Dominant Meaning Word Association Responses (Score) and Observed Priming in Each Part of Experiment 2

\begin{tabular}{|c|c|c|c|}
\hline \multicolumn{2}{|c|}{ Prime History } & Score & Priming \\
\hline \multicolumn{4}{|c|}{ Part 1} \\
\hline $\begin{array}{r}\text { Prior } \\
- \\
-\end{array}$ & $\begin{array}{l}\text { Current } \\
\text { Dominant } \\
\text { Secondary }\end{array}$ & $\begin{array}{l}.776 \\
.440\end{array}$ & $\begin{array}{l}.171 \\
.165\end{array}$ \\
\hline \multicolumn{4}{|c|}{ Part 2} \\
\hline Prior & Current & & \\
\hline Dominant & - & .676 & .071 \\
\hline Secondary & - & .510 & .095 \\
\hline Secondary & Dominant & .601 & -.004 \\
\hline Dominant & Secondary & .583 & .022 \\
\hline \multicolumn{4}{|c|}{ Part 3 Unprimed } \\
\hline Part 1 & Part 2 & & \\
\hline Dominant & - & .680 & $.075^{*}$ \\
\hline Secondary & - & .504 & .101 \\
\hline Dominant & Secondary & .633 & .028 \\
\hline Secondary & Dominant & .545 & .060 \\
\hline
\end{tabular}

Note-Priming is the deviation from the normative baseline of .615 . * Priming in Part 3 is defined with respect to the Part 1 prime for all conditions.

tionship to the homographs, we restrict our reporting of results to the effectiveness of the repetition conditions. The initial priming direction interacted both with occurrence $\left[F(2,216)=67.20, M S_{e}=0.016\right]$ and with prime history $\left[F(1,108)=21.47, M S_{\mathrm{e}}=0.017\right]$. There were no significant main effects of occurrence or prime history and no interaction between the two.

However, these effects must be interpreted in light of the significant three-way interaction between occurrence, prime history, and initial prime direction $[F(2,216)=6.23$, $\left.M S_{\mathrm{e}}=0.016\right]$. In the control condition (unrelated context on second occurrence), the effect of the initial prime was reduced on the second occurrence as control item responses shifted toward the norm and then remained unchanged on the third occurrence. In the mixed condition, the alternate meaning was successfully primed on the second occurrence. Further, on the third occurrence, performance moved back toward the first-occurrence mean.

A second ANOVA examined the primacy effect based on initial homograph meaning. As shown in Table 2, the means for the mixed items moved in different directions from the second to the third occurrence of the homograph based on initial meaning primed. Means for homographs initially primed in the dominant direction moved toward the dominant meaning, while means for homograph initially primed in the secondary direction changed in that direction. A 2 (dominant, secondary prime) $\times 2$ (second or third occurrence) ANOVA was performed to see whether this observed effect was significant. The significant interaction $\left[F(1,108)=15.32, M S_{\mathrm{e}}=0.026\right]$ indicates that responses to the unprimed third occurrences of homographs in the mixed condition are in the direction of the initial priming experience.
We had hypothesized that the longer the delay between the second and third occurrences of the homograph, the greater the change toward the initially primed meaning. The hypothesis was not supported; the change toward the initial prime was .06 in the Lag 3 delay condition, while the corresponding change in the Lag 20 condition was .04.

Neither the effect of the lag between first and second occurrences nor the delay prior to testing the third occurrence in the mixed prime history condition had a significant effect on performance. Nevertheless, in the control condition the effect of the prime declined significantly (.09) from first occurrence to the neutral primed second occurrence. In the mixed condition, there was a similar decline of second prime effectiveness $(.05)$ relative to the neutral context third occurrence. It must be concluded that decline was relatively rapid at a lag of 3 or fewer items. A possible explanation of the rapid change is that a portion of the immediate priming effect depends on the $N-1$ prime being in the active state. Like the "automatic priming" of Posner and Snyder (1975), that activation may decay in a matter of seconds or be disrupted by another word presentation. In that event, the prior prime would be inactive before the next occurrence of a homograph even at short lags.

In the mixed condition on second occurrences, .583 of the responses were in the dominant direction despite the presence of a secondary meaning prime. Responses to the dominant primed condition were near baseline at .601 . These data are compatible with the "inhibition of competing meanings" hypothesis of Simpson and Kang (1994). Alternatively, it might be argued that computing priming from the normative baseline is misleading because the first-occurrence prime produces a temporary shift in the normative frequency of the homograph meanings, leading to an underestimate of the amount of priming. The results with respect to second occurrences in Experiment 2 speak directly to this point. As shown in Table 3, computations of priming based on the control condition, which reflect any change in baseline, do not support this explanation. The amount of priming in relation to the appropriate control was .09 . This value was slightly more than half of the priming obtained (.17) for the first-occurrence prime. Some form of inhibition of alternative meanings results from the processing of a homograph within a specific semantic context.

Table 3

Effect of Context on Second-Occurrence Responses to Homographs (Score): Change in the Direction of Second-Occurrence Prime Event

\begin{tabular}{|c|c|c|c|c|c|c|}
\hline \multirow{3}{*}{ First } & & \multicolumn{5}{|c|}{ Occurrence } \\
\hline & & \multicolumn{2}{|c|}{ Second Mixed } & \multicolumn{2}{|c|}{ Second Neutral } & \multirow[b]{2}{*}{ Difference } \\
\hline & Score & Score & Change & Score & Change & \\
\hline Dominant & .78 & .59 & .19 & .68 & .10 & .09 \\
\hline Secondary & .44 & .60 & .16 & .51 & .07 & .09 \\
\hline
\end{tabular}


Finally, we note that performance on the mixed-prime items of this experiment were highly similar to those in Experiment 1, despite the many differences in method.

\section{EXPERIMENT 3}

In both Experiments 1 and 2, the proportion of responses related to the meaning elicited by a single priming context declined when the homograph was later presented in a neutral context. Moreover, mean responses in the primed meaning class appeared to asymptote, indicating a continued influence of the prime. In Experiment 3 we examined whether that asymptote might be altered by a second priming experience of the same semantic meaning as the first.

Method
Participants
Thirty-two Adelphi undergraduate students participated in the
experiment in partial fulfillment of a class requirement.
Materials
Eight lists were created using the same 28 homographs, with four
primes selected for each homograph, two for each meaning. An ad-
ditional 56 filler items (words with only one meaning) were included
as part of the 280 -item list.
The lists were divided into three parts. In Part 1 (Trial $1-112$ ), 14
of the homographs were preceded by a dominant meaning prime
and 14 with a secondary meaning prime. The prime-homograph se-
quences were randomized along with the 56 filler words. In Part 2
(Trials $113-218$ ), the homographs were presented again. Of the 14
homographs primed with the dominant meaning in Part 1,7 were
primed with a different associate to the dominant meaning and 7 with
a secondary prime. Similarly, of the 14 homographs primed with a
secondary meaning in Part 1,7 were primed with another secondary
meaning word and 7 with a dominant meaning prime. This assign-
ment of primes to the homographs resulted in two conditions, the
same-context condition and the mixed-context condition. Filler
words were repeated in Part 2 and randomly interspersed with the
prime-homograph sequences. In Part 3 (Trials $219-280$ ), the
28 homographs were presented unprimed and randomly mixed with
34 filler words.

\section{Procedure}

The procedure was the same as that of Experiment 1, with a maximum of $6 \mathrm{sec}$ for each trial. A list consisted of 280 word association trials. Approximately $20-25$ min were required to complete the task.

\section{Results and Discussion}

The scoring procedure was identical to that of the other experiments. Table 4 summarizes the results.

An analysis of Part 1 performance indicated a significant effect of the prime $\left[F(1,31)=101.24, M S_{\mathrm{e}}=\right.$ 0.0235]. An ANOVA of Part 2 was performed in which the factors of the within-subjects design were two levels of Part 1 prime (dominant, secondary) $\times$ two levels of Part 2 prime (dominant, secondary). Both the effect of Part 1 prime $\left[F(1,31)=15.36, M S_{\mathrm{e}}=0.0346\right]$ and Part 2 prime $\left[F(1,31)=64.76, M S_{\mathrm{e}}=0.205\right]$ were significant. The interaction of the two priming events did not reach significance $\left(F<1, M S_{\mathrm{e}}=0.0379\right)$. As in Experiments
Table 4

Proportion of Dominant Meaning Word Association Responses (Score) and Observed Priming in Each Part of Experiment 3

\begin{tabular}{|c|c|c|c|}
\hline \multicolumn{2}{|c|}{ Prime History } & Score & Priming \\
\hline \multicolumn{4}{|c|}{ Part 1} \\
\hline Prior & Current & & \\
\hline- & Dominant & .792 & .187 \\
\hline- & Secondary & .467 & .138 \\
\hline \multicolumn{4}{|c|}{ Part 2} \\
\hline Prior & Current & & \\
\hline Dominant & Dominant & .835 & .230 \\
\hline Secondary & Secondary & .438 & .167 \\
\hline Secondary & Dominant & .629 & .024 \\
\hline Dominant & Secondary & .545 & .060 \\
\hline \multicolumn{4}{|c|}{ Part 3 Unprimed } \\
\hline Part 1 & Part 2 & & \\
\hline Dominant & Dominant & .768 & $.163^{*}$ \\
\hline Secondary & Secondary & .455 & .150 \\
\hline Dominant & Secondary & .665 & .060 \\
\hline Secondary & Dominant & .581 & .024 \\
\hline
\end{tabular}

Note-Priming is the deviation from the normative baseline of .615 . *Priming in Part 3 is defined with respect to the Part 1 prime for all conditions.

1 and 2, a prime of different meaning was less effective than the initial priming manipulation.

In Part 3 the homographs were unprimed. We performed two ANOVAs contrasting the performance in Parts 2 and 3 separately for the conditions that had received different meaning primes in Parts 1 and 2 (mixed prime history) and the conditions that had same meaning primes in Parts 1 and 2 (unmixed prime history). Each ANOVA therefore was a 2 (Part 2, Part 3 ) $\times 2$ (dominant, secondary) prime type in Part 2, within-subject design.

Consistent with the results for the mixed prime history items in Experiments 1 and 2, we obtained a crossover interaction indicating a "primacy effect." Part 2 performance was in the direction of the Part 2 prime, but Part 3 performance was in the direction of the Part 1 prime $\left[F(1,31)=9.24, M S_{\mathrm{e}}=0.0184\right]$. No other effect approached significance.

In the unmixed prime history condition, there was only an effect of the Part 2 prime $\left[F(1,31)=58.18, M S_{\mathrm{e}}=\right.$ $0.0485]$. In this ANOVA a decay in the effectiveness of the prime would have been shown by a part $\times$ prime interaction since decay was toward the baseline. That interaction fell short of significance $\left[F(1,31)=2.83, M S_{\mathrm{e}}=\right.$ $0.0143, p>.10]$. The observed decline in priming from Part 2 to Part 3 was .042-about half the decline observed in the single-prime conditions in Experiments 1 and 2. Two priming events in the same direction increased priming slightly and resulted in a more stable selection of the primed meaning of the homograph.

\section{GENERAL DISCUSSION}

The major purpose of the experiments reported was to test the hypothesis that an attempt to elicit a response to 
a homograph consistent with a semantic context would be sensitive to the prior exposure of that homograph in the WAT. We were interested in meaning selection, not in lexical access. ${ }^{3}$ The meaning selected in the mixed-context conditions clearly indicated a significant decrement in the effect of semantic context. In short, the discrete response data of the WAT paralleled the reaction time results in priming studies that led Simpson and Kang (1994) to formulate the "inhibition of competing meanings" hypothesis.

The Simpson and Kang (1994) view was developed employing word naming, an "online" task. In the WAT, the participant is free to choose a response from whatever comes to mind. However, meaning selection is the ultimate "name of the game" in real-life communication, and it is interesting that the effects in word selection correspond to the effects that are observed in online tasks. It makes sense to us that the associative process mirrors a process that Simpson and Kang feel is important to language processing, since conversation involves not only understanding that which the other is saying, but also stating one's own ideas in a way that promotes communication. Our experiments suggest that the lexicon might be led by the effects of homograph processing to avoid emitting words not appropriate to the discourse context.

In Experiment 2, we rejected the hypothesis that priming in the WAT merely changed the local homograph baseline. Some form of interference with the effectiveness of alternative primes in the WAT was clearly required. We therefore adopted the view proposed by Simpson and Kang (1994) that the result was due to some form of "inhibition of competing meanings."

In our view, the "inhibition of competing meanings" can be understood as the inhibition of competing associates to those elicited by the primed homograph. This interpretation is offered in contrast to an interpretation that renders the phrase "inhibition of competing meanings" to mean a cutting off of the pathways between the lexical representation of the homograph and one set of its associations.

To explain this conclusion we need to briefly describe the experiments with respect to homographs priming related words in the naming task (Simpson \& Kang, 1994; Simpson \& Kellas, 1989). If the effect of a first-occurrence prime trial (e.g., BANK as a prime for the naming of the target word SAVINGS) was simply to disconnect BANK from its alternate meaning of "embankment," the result would be to in effect make the word BANK unrelated to words like RIVER. In that case an attempt to prime the target RIVER with the prime BANK would be no different from attempting to prime RIVER with the word MARCH, a homograph unrelated to RIVER. In their studies the different meaning context condition results in reaction times longer than those of an unrelated control condition. Hence it is the associate that is inhibited in the presence of the repeated homograph.

A detailed model of inhibition applied to the WAT must consider both the persistence of the inhibitory effect over a large number of intervening events and the partial recovery of the inhibited associations when the homograph is presented in a neutral context. In addition, the "primacy effect" observed in response to the unprimed homograph after that homograph has been exposed to a mixed prime history needs to be explained.

Our explanation of these results employs a theoretical extension of the Simpson and Kang (1994) hypothesis. We adopt the idea of a suppression of competing associates when a meaning is selected for a homograph (Gernsbacher, 1990; Neill, 1989). Employing a stimulus sampling framework (Estes, 1950), we propose to account for the "active and specific inhibition of competing meanings" as a case of "conditioned suppression" of associates inappropriate to the context. We propose that the suppression response is connected with, or conditioned to, a sample of the active elements of the homograph and a sample of the elements of the environmental context. In this view the probability of a suppression response to a subsequent occurrence of a homograph depends on the proportion of elements in the stimulus sample at homograph occurrence that are connected to the inhibitory mechanism. After a single priming event, only some elements of the homograph's sample universe will be conditioned to the inhibitory response. The stimulus sample at the later occurrence of the homograph is unlikely to be the same as that at the time of learning, hence the suppression response might not occur. If we assume that some of the activated elements of the prime are part of the original conditioned sample and that these elements rapidly decay, we can account for the observed rapid change toward baseline in a neutral context. A stimulus sampling view also implies that more than one presentation of a homograph in the same semantic context will lead to greater stability of the inhibitory response, since the number of contextual elements conditioned to the suppression response would be increased by additional learning trials.

From a theoretical perspective, environmental contextual stimuli need to be included in the stimulus sample as a mechanism to ensure that the inhibitory response is situationally specific. In their absence, the theory would predict that secondary associates of homographs would tend to be inhibited in new contexts. Such a prediction would be inconsistent with the results for new contexts in the homograph literature as well as first occurrences in these experiments.

An explanation of the primacy effect requires additional mechanisms. Anderson $(1981,1996)$ has suggested that primacy effects are often attributable to differences in attentional processes. In the case of the WAT, it may be that the process of trying to produce a response in the presence of the inhibitory tendency is more effortful. Such an attentional demand would result in fewer attentional resources devoted to the suppression and consequent inhibition of incompatible associations. As a result of sampling, some of the conditioned inhibitory elements from the first-occurrence event are not in the second event sample. These elements remain attached to the initial inhibitory response. Due to the attentional problem, fewer of the elements in the second-occurrence sam- 
ple would be conditioned to suppressing associates of the first-occurrence meaning. Therefore at the time of next testing the probability that the first-occurrence inhibitory elements dominate the stimulus sample would be higher than the probability of the second-occurrence inhibitory elements dominating. The observed primacy effect is an outcome consistent with the explanation offered.

Finally, it is not unreasonable to expect that within an experimental context these inhibitory changes would persist. Therefore, it would not be unreasonable to expect a transfer of an inhibitory (conditioned suppression) effect from one homograph priming task to another. Work is under way to test this implication of the conditioned suppression view.

\section{REFERENCES}

ANDERSON, N. H. (1981). Foundations of information integration the ory. New York: Academic Press.

ANDERSON, N. H. (1996). A functional theory of cognition. Mahwah, $\mathrm{NJ}$ : Erlbaum.

Balota, D. A. (1990). The role of meaning in word recognition. In D. A. Balota, G. B. Flores d'Arcais, \& K. Rayner (Eds.), Comprehension processes in reading (pp. 9-32). Hillsdale, NJ: Erlbaum

Berger, S. A., BubKa, A., \& Gorfein, D. S. (1990, June). Prior contextual priming effects on word-associations to homographs. Poster presented at the annual meeting of the American Psychological Society, Dallas.

Clifton, C., JR. (1966). Some determinants of priming word associations. Journal of Verbal Learning \& Verbal Behavior, 5, 167-171.

COFER, C. N. (1967). Conditions for the use of verbal associations. Psychological Bulletin, 68, 1-12.

Cramer, P. (1968). Mediated priming of polysemous stimuli. Journal of Experimental Psychology, 78, 137-144.

Estes, W. K. (1950). Toward a statistical theory of learning. Psychological Review, 57, 94-107.

GeRNSBACHER, M. A. (1990). Language comprehension as structure building. Hillsdale, NJ: Erlbaum.

Glanzer, M., \& CUNITZ, A. R. (1966). Two storage mechanisms in free recall. Journal of Verbal Learning \& Verbal Behavior, 5, 351-360.

Gorfein, D. S., Viviani, J. M., \& LedDo, J. (1982). Norms as a tool for the study of homography. Memory \& Cognition, 10, 503-509.

GorfEIN, D. S., \& WALTERS, M. F. (1989). When does "soar" become "sore"? Some comments on the chapter of Simpson and Kellas. In D. S. Gorfein (Ed.), Resolving semantic ambiguity (pp. 57-62). New York: Springer-Verlag.

KrUEGer, M. A. (1990). Sentence priming effects on the processing of ambiguous words. Unpublished master's thesis, University of Nebraska, Omaha.

KUČERA, M., \& FRANCIS, W. (1967). Computational analysis of presentday American English. Providence, RI: Brown University Press.
Myers, J. L., \& WeLl, A. D. (1991). Research design and statistical analysis. New York: HarperCollins.

NEILL, W. T. (1989). Lexical ambiguity and context: An activationsuppression model. In D. S. Gorfein (Ed.), Resolving semantic ambiguity (pp. 63-83). New York: Springer-Verlag.

ONIFER, W., \& SWINNEY, D. A. (1981). Accessing lexical ambiguities during sentence comprehension: Effects of frequency of meaning and contextual bias. Memory \& Cognition, 9, 225-236.

PoSNer, M. I., \& SNYDER, C. R. R. (1975). Attention and cognitive control. In R. L. Solso (Ed.), Information processing and cognition: The Loyola Symposium (pp. 55-85). Hillsdale, NJ: Erlbaum

SChVANEVELDT, R. W., MEYER, D. E., \& BeCKER, C. A. (1976). Lexical ambiguity, semantic context, and visual word recognition. Journal of Experimental Psychology: Human Perception \& Performance, 2, 243256.

SEGAL, S. J. (1967). Priming of associative test responses by differential verbal contexts. Journal of Experimental Psychology, 74, 370-377.

SEGAL, S. J., \& CoFER, C. N. (1960). The effects of recency and recall on word association [Abstract]. American Psychologist, 13, 451

SimPSON, G. B., \& KANG, H. (1994). Inhibitory processes in the recognition of homograph meanings. In D. Dagenbach \& T. H. Carr (Eds.), Inhibitory mechanisms in attention, memory, and language (pp. 359381). New York: Academic Press.

Simpson, G. B., \& Kellas, G. (1989). Dynamic conceptual processes and lexical access. In D. S. Gorfein (Ed.), Resolving semantic ambiguity (pp. 40-56). New York: Springer-Verlag.

STORMS, L. H. (1958). Backward associations: A situational effect. Journal of Experimental Psychology, 55, 390-395.

WALTERS, M. F. (1988). The priority effect in implicit memory. Unpublished doctoral dissertation, Adelphi University, Garden City, NY.

\section{NOTES}

1. In a check on these norms, we tested 20 Adelphi University students on a similar list, but re-paired the homographs and their primes to form unrelated pairs. In that study mean performance for each of the homograph subgroups did not differ significantly from the values reported by Gorfein et al. (1982).

2. All ANOVAs included the delay variable as a between-groups variable even though the delay occurred between Parts 2 and 3 of the list. This was done to check on the comparability of groups assigned to the different delays and resulted in the degrees of freedom for the ANOVA being $n-2$ rather than $n-1$. The effect of delay never approached statistical significance and is not discussed in the text.

3 . We believe that the question of what is accessed at the magic moment of access may be an intractable one (see Balota, 1990, for a discussion of this issue). We therefore have chosen to focus our discussion on the observable event-the word chosen as a response.

(Manuscript received September 8, 1998; revision accepted for publication August 23, 1999.) 